\title{
Formation control of VTOL UAV vehicles under switching-directed interaction topologies with disturbance rejection
}

\section{Meisam Kabiri, Hajar Atrianfar \& Mohammad B. Menhaj}

To cite this article: Meisam Kabiri, Hajar Atrianfar \& Mohammad B. Menhaj (2018) Formation control of VTOL UAV vehicles under switching-directed interaction topologies with disturbance rejection, International Journal of Control, 91:1, 33-44, DOI: 10.1080/00207179.2016.1266518

To link to this article: http://dx.doi.org/10.1080/00207179.2016.1266518

Accepted author version posted online: 29

Nov 2016.

Published online: 22 Dec 2016.

Submit your article to this journal $\pi$

Џ Article views: 71

Q View related articles $\sqsubset$

View Crossmark data $\nearrow$

Citing articles: 1 View citing articles $ک$ 


\title{
Formation control of VTOL UAV vehicles under switching-directed interaction topologies with disturbance rejection
}

\author{
Meisam Kabiri, ${ }^{a, b}$, Hajar Atrianfar ${ }^{a}$ and Mohammad B. Menhaj ${ }^{a, b}$

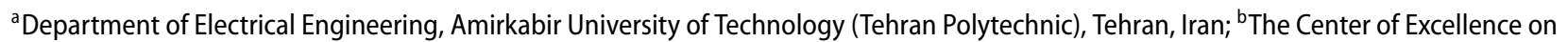 \\ Control and Robotics, Department of Electrical Engineering, Amirkabir University of Technology (Tehran Polytechnic), Tehran, Iran
}

\begin{abstract}
This paper addresses the adaptive formation control of a group of vertical take-off and landing (VTOL) unmanned aerial vehicles (UAV) with switching-directed interaction topologies. In addition, to tackle the adverse effect of disturbances, a couple of smooth bounded estimators are involved in the procedure design. Exploiting an extraction algorithm, we take advantage of the fully actuated rotational dynamics, to control the translational dynamics of each vehicle. We propose a distributed control scheme such that all vehicles track a desired reference velocity signal while keeping a desired prespecified formation. In this framework, the underlying topology of the agents may switch among several directed graphs, each having a spanning tree. The stability of the overall closed-loop system is proved through Lyapunov function. Finally, simulation results are given to better highlight the effectiveness of the proposed control scheme.
\end{abstract}

\section{ARTICLE HISTORY}

Received 16 May 2016

Accepted 26 November 2016

\section{KEYWORDS}

Formation control; disturbance rejection; VTOL aircraft; time-varying topologies; underactuated systems

\section{Introduction}

Cooperation of multiple agents, compared to a single, renders several advantages such as lower cost, higher efficiency, more flexibility and more robustness. Complex tasks can also be done by a system of multiple agents. In one of these tasks, formation control, agents form a specific geometric shape merely relying on information from their neighbouring agents.

Thanks to these benefits and some important features of vertical take-off and landing (VTOL) unmanned aerial vehicles (UAVs) like high maneuverability and hovering flight, the motion coordination of this class of systems has drawn much interest in recent years and has been utilised in several applications in areas such as surveillance and reconnaissance, search and rescue mission, monuments inspection (Kopfstedt, Mukai, Fujita, \& Ament, 2008; Nigam, Bieniawski, Kroo, \& Vian, 2012; Pack, DeLima, Toussaint, \& York, 2009).

Motion equations of VTOL UAVs are generally governed by the fully actuated rotational dynamics with a three-dimensional vector input and the translational dynamics with a three-degree-of-freedom and just with a one-dimensional input thrust which accelerates the aircraft along the $z$-axis of the body frame. Attitude control of this type of systems, as a rigid body, has been widely studied and many results have been reported (see, for instance, Abdessameud, Tayebi, \& Polushin, 2012;
Erdong \& Zhaowei, 2009; Hu \& Zhang, 2015; Ren, 2006; Tayebi, 2008).

Yet, controlling the position poses more difficulties because of underactuation of the translational dynamics, especially when it comes to a coordination framework. To deal with this challenge, several methods have been developed (Abdessameud \& Tayebi, 2009, 2011, 2013; Cabecinhas, Cunha, \& Silvestre, 2014; Lee, 2012; Pflimlin, Soueres, \& Hamel, 2007, 2004; Roberts \& Tayebi, 2011). In Lee (2012), by extending the passive decomposition method, a distributed back-stepping control framework for coordination of multiple thrust-propelled vehicles is proposed. With an aid of extraction-based method and separation of the rotational dynamics from the translational dynamics, a position controller for trajectory tracking for a single VTOL with external disturbances was presented in Roberts and Tayebi (2011). In Abdessameud and Tayebi $(2009,2011,2013)$, with a similar method, the formation of a group of VTOLs was studied. In Cabecinhas et al. (2014), based on back-stepping method, an adaptive controller for a single underactuated thrustpropelled vehicle to track a predefined spatial path was fully developed.

Communicating neighbouring agents play a vital role in cooperation of multiple agents. As in real-world applications, the environment in which agents communicate is not perfect and some obstacles may appear between agents, some new communication links may be created 
and some may be failed which leads to the variation of network topologies. However, to the authors' best knowledge, no studies have been found considering cooperation of VTOL UAVs under switching interaction topologies. Several publications have appeared in recent years investigating coordination control of first- and secondorder system models with switching topologies (Atrianfar \& Haeri, 2015; Lin \& Jia, 2010; Olfati-Saber \& Murray, 2004; Atrianfar \& Haeri, 2012 ; Qin, Gao, \& Zheng, 2011; Ren \& Beard, 2008; Zheng \& Wang, 2012; Atrianfar \& Haeri, 2013). For instance, in Olfati-Saber and Murray (2004), the consensus problem for multiple first-order systems was established with strongly balanced digraphs. In Atrianfar \& Haeri (2012), a larger class of digraphs including balanced ones is introduced which leads to consensus in networks of agents with first-order dynamics. In Ren and Beard (2008), some results for coordination for systems of first- and second-order dynamics under some improved conditions on topology networks can be found. Qin et al. (2011) investigated the consensus of second-order systems with balanced graphs and arbitrarily switching signal. With weaker conditions on network topologies, in Lin and Jia (2010) and Zheng and Wang (2012) and Atrianfar and Haeri (2013), consensus problem for second-order dynamics, respectively, under jointly connected and jointly weakly connected graphs, was investigated. Atrianfar and Haeri (2015) extended the existing results to the case of consensus reaching in second-order dynamics under switching topologies and time-varying communication delays. However, there still remains much to be done to develop methods for motion control of complex, underactuated systems with nonlinear coupling and, as it will become clear throughout the paper, these results cannot be extended straightforwardly to our case due to input constraints on the translational input.

Exploiting the extraction algorithm, the translational dynamics of VTOL UAVs is transformed to a fully actuated second-order system with a new input and a perturbation term. In other words, the problem is converted to cooperation of multiple second-order perturbed system and trajectory-tracking problem for nonlinear rotational dynamics. The difficulty arising in extending studies on double integrators to this case, is some constraints on the new input imposed by the extraction algorithm. This input should be smooth enough and bounded in advance. This scenario can be even more challenging when the impact of disturbances are involved.

The main motivation of this paper is to extend the results in Abdessameud and Tayebi (2013) to the case where the underlying network topology changes over time under a pair of bounded disturbances affecting both the translational and rotational dynamics. Using extraction algorithm, auxiliary systems, virtual agents along with designing a suitable controller for attitude dynamics enables us to extend the cooperative results for linear systems to our nonlinear case. In the procedure design, we designate a second-order virtual agent to each aircraft and make them achieve the cooperation task that we expect from the actual ones. Then, by guaranteeing that states of the actual vehicle converge to its corresponding virtual states, reaching the overall goal is provided. This approach along with introducing some auxiliary systems helps us to overcome the major constraint on input, i.e. twice differentiability. Moreover, to overcome the another constraint, boundedness, we make use of the saturation function and the projection operator.

The rest of the paper is organised as follows. Preliminaries are given in the following section. The formation problem is formulated in Section 3. The controllers for the translational and rotational dynamics are introduced, respectively, in Sections 4 and 5. The main result and the convergence analysis for the proposed controllers are provided in Section 6. Numerical simulations and our conclusion are presented in Sections 7 and 8, respectively.

\section{Preliminaries}

\subsection{Notation}

Throughout this paper, $\|$.$\| denotes the Euclidean norm,$ $I_{3} \in \mathbb{R}^{3 \times 3}$ denotes the identity matrix. $S(x): \mathbb{R}^{3} \rightarrow \mathbb{R}^{3 \times 3}$ denotes the skew-symmetric matrix such that

$$
\forall x=\left(\begin{array}{l}
x_{1} \\
x_{2} \\
x_{3}
\end{array}\right) \in \mathbb{R}^{3}: S(x)=\left(\begin{array}{ccc}
0 & -x_{3} & x_{2} \\
x_{3} & 0 & -x_{1} \\
-x_{2} & x_{1} & 0
\end{array}\right) .
$$

Let $\hat{x}$ denote the estimation of unknown disturbance $x$. $\operatorname{Re}\{x\}$ and $\operatorname{Im}\{x\}$, respectively, denote the real and imaginary part of the $x$.

A function $\chi(x)=\left(\varrho\left(x_{1}\right), \varrho\left(x_{2}\right), \varrho\left(x_{3}\right)\right) \rightarrow \mathbb{R}^{3}$ is a differentiable saturation function with $\varrho: \mathbb{R} \rightarrow \mathbb{R}$ and properties:

- $\frac{d \varrho(x)}{x}$ is bounded for all $\mathrm{x}$,

- $|\varrho(x)|<M$ for all $x$,

- $\mathrm{\varrho}(0)=0$ and $x \varrho(x)>0$.

\subsection{Graph theory}

Information exchange among the agents can be represented by a graph. Let the graph $\mathrm{G}$ consist of the node set $\mathcal{V}=\{1, \ldots, n\}$, a set of edges $\mathcal{E} \in\{\mathcal{V} \times \mathcal{V}\}$ and an adjacency matrix $A=\left[a_{i j}\right] \in \mathbb{R}^{n \times n}$. We consider vehicles as nodes of a graph and communication links as edges of a graph. The weighted adjacency matrix $A$ is defined such that $a_{i j}>0$ if $(i, j) \in \mathcal{E}$, while $a_{i i}=0$. An edge $(i, j)$ 
indicates that the vehicle $j$ is a neighbour of vehicle $i$ and the $i$ th vehicle has access to information of the $j$ th vehicle. The Laplacian matrix of a weighted graph $A$ is defined as $\mathcal{L}=\left[l_{i j}\right] \in \mathbb{R}^{n \times n}$, where $l_{i i}=\sum_{j} a_{i j}$ and $l_{i j}=-a_{i j}$. We assume each agent can communicate with its neighbours and the interaction topology between agents is directed and changes over time. To describe the varying topologies, we make use of a piecewise right-continuous switching function $\sigma(t):[0, \infty] \rightarrow \mathcal{P}=\{1,2, \ldots, N\}$, where $N$ is the total number of all possible directed communication graphs. $G^{\sigma(t)}$ denotes the communication graph at time $t$ and $\mathcal{L}^{\sigma(t)}$ is the corresponding Laplacian matrix. We assume that every possible graph topology contains a directed spanning tree. A directed spanning tree is a directed graph containing all nodes in which there exists a vertex called the root such that there is a directed path from every other node to this node. We say a graph contains a spanning tree if a subset of edges forms a directed spanning tree.

We assume that there exists an infinite sequence of non-overlapping time intervals $\left[t_{k}, t_{k+1}\right], k=1,2, \ldots$, with $t_{0}=0$ and $\tau_{1}>t_{k+1}-t_{k}>\tau_{0}>0$ for some positive constants $\tau_{0}$ and $\tau_{1}$, where $\tau_{0}$ is called the dwell time and the communication graph is assumed to be fixed on each time interval.

\subsection{System model}

Consider $n$ as the number of vehicles. The motion equation of the aircraft $i$ modelled as a rigid body can be simplified as

$$
\begin{gathered}
\left\{\begin{array}{l}
\dot{p}_{i}=v_{i}, \\
\dot{v}_{i}=g \hat{z}-\frac{\mathcal{T}_{i}}{m_{i}} R\left(Q_{i}\right)^{T} \hat{z}+b_{i},
\end{array}\right. \\
\left\{\begin{array}{c}
\dot{Q}_{i}=\frac{1}{2}\left(\begin{array}{c}
\eta_{i} I_{3}+S\left(q_{i}\right), \\
-q_{i}^{T}
\end{array}\right) \omega_{i}, \\
J_{i} \dot{\omega}_{i}=\Gamma_{i}-S\left(\omega_{i}\right) J_{i} \omega_{i}+S(\hat{z}) R\left(Q_{i}\right) d_{i},
\end{array}\right.
\end{gathered}
$$

where $v_{i} \in \mathbb{R}^{3}$ and $p_{i} \in \mathbb{R}^{3}$ are, respectively, the linear velocity and the position of the $i$ th aircraft described in the inertial frame. $b_{i}$ and $d_{i}$ are the constant disturbances affecting the translational and rotational dynamics of aircraft $i$ in which $b_{i}=\frac{1}{m} F_{\text {ext }}$ and $d_{i}=\varepsilon_{M} F_{\text {ext }}$, where $F_{\text {ext }}$ is the constant inertial referenced disturbance and $\varepsilon_{M}$ is the lever arm that creates a disturbance torque due to $F_{\text {ext }}$. The parameterisation of disturbances is taken from Roberts and Tayebi (2011) and Pflimlin et al. (2007) in which it is assumed that the aerodynamic effects $F_{\text {ext }}$ are applied at some point on the body-referenced $\mathrm{z}$-axis and at the distance $\varepsilon_{M}$ away from the vehicle center of gravity. $m_{i}$ and $g$ are the mass and the gravitational acceleration of the $i$ th vehicle and $\hat{z}=(0,0,1)^{T} . J_{i} \in \mathbb{R}^{3 \times 3}$ is the moment of inertia of the $i$ th aircraft with respect to its body-fixed frame. The scalar $\mathcal{T}_{i}$ and the vector $\Gamma_{i} \in \mathbb{R}^{3}$ are the thrust input and control torque input for the $i$ th aircraft, respectively, for translational and rotational dynamics. $\omega_{i}$ denotes the body-referenced angular velocity of the $i$ th vehicle. To represent the attitude, we use the singularfree unit quaternion which also has ambiguity (for more information, see Diebel, 2006; Shuster, 1993). The unit quaternion $Q_{i}=\left(q_{i}^{T}, \eta_{i}\right)^{T}$, composed of the vector part $q_{i} \in \mathbb{R}^{3}$ and the scalar part $\eta_{i} \in \mathbb{R}$, represents the orientation of the $i$ th vehicle's body frame with respect to the inertial frame which satisfies the constraint $q_{i}^{T} q_{i}+$ $\eta_{i}^{2}=1$. The inverse of unit quaternion $Q_{i}$ is defined as $Q_{i}^{-1}=\left(-q_{i}^{T}, \eta_{i}\right)^{T}$ with the quaternion identity given by $Q_{I}=(0,0,0,1)^{T}$. The unit quaternion multiplication is defined by $Q_{i} \odot Q_{j}=\left(\left(q_{i} \eta_{j}+q_{j} \eta_{i}+S\left(q_{i}\right) q_{j}\right)^{T}, \eta_{i} \eta_{j}-\right.$ $\left.q_{i}^{T} q_{j}\right)^{T}$ which is also a unit quaternion, where $S(x)$ is the skew-symmetric matrix. The rotation matrix $R\left(Q_{i}\right)$ which brings the inertial frame into the body frame is obtained by $R\left(Q_{i}\right)=\left(\eta_{i}^{2}-q_{i}^{T} q_{i}\right) I_{3}+2 q_{i} q_{i}^{T}-2 \eta_{i} S\left(q_{i}\right)$.

\section{Problem formulation}

Our objective is to force all vehicles to track a bounded desired reference velocity signal $v_{d}(t)$ available to all vehicles while maintaining inter-vehicle stationary formation pattern, that is, $v_{i}(t) \rightarrow v_{d}(t)$ and $p_{i}-p_{j} \rightarrow \delta_{i j}$, where $\delta_{i j}=\delta_{i}-\delta_{j}$ and $\delta_{i}$ is the desired offset position of the $i$ th aircraft with respect to the centre of the formation. This should be achieved regarding the switching-directed information exchange between the aircrafts

Assumption 3.1: The first, second and third timederivatives of $v_{d}(t)$ are bounded and $\left\|\dot{v}_{d}\right\|<g$.

Due to the underactuation of the translational dynamics, it is required to use the orientation of the aircraft to control its position. We can put Equation (1) into the form

$$
\left\{\begin{array}{l}
\dot{p}_{i}=v_{i} \\
\dot{v}_{i}=F_{i}+\tilde{F}_{i}+b_{i}
\end{array}\right.
$$

with

$$
\begin{aligned}
& F_{i}=g \hat{z}-\frac{\mathcal{T}_{i}}{m_{i}} R\left(Q_{d_{i}}\right)^{T} \hat{z}, \\
& \tilde{F}_{i}=\frac{\mathcal{T}_{i}}{m_{i}}\left(R\left(Q_{d_{i}}\right)^{T}-R\left(Q_{i}\right)^{T}\right) \hat{z},
\end{aligned}
$$

where $\tilde{F}_{i}$ is the underactuation error and $F_{i}$ is the intermediary control input to be designed for the translational dynamics. Then, based on $F_{i}$, the appropriate thrust input $\mathcal{T}_{i}$ and attitude $Q_{d_{i}}$ should be determined. To do so, several extraction algorithms have been presented in the literature (Hua, Hamel, \& Samson, 2013; Roberts \& Tayebi, 2011; Roza \& Maggiore, 2014). In this work, we make use 
of the one introduced in Abdessameud and Tayebi (2013) which is restated in the following Lemma.

Lemma 3.1. Let $F=\left(\mu_{1}, \mu_{2}, \mu_{3}\right)^{T}$. With respect to Equations (1)-(5), the magnitude thrust $\mathcal{T}$ and the desired orientation $Q_{d}=\left(q_{d}^{T}, \eta_{d}\right)^{T}$ can be obtained by

$$
\begin{aligned}
\mathcal{T} & =m\|g \hat{z}-F\|, \\
\eta_{d} & =\sqrt{\frac{1}{2}+\frac{m\left(g-\mu_{3}\right)}{2 \mathcal{T}}}, \quad q_{d}=\frac{m}{2 \mathcal{T} \eta_{d}}\left(\begin{array}{c}
\mu_{2} \\
-\mu_{1} \\
0
\end{array}\right) .
\end{aligned}
$$

This extraction will be always well defined if

$$
F \neq g \hat{z}
$$

See Abdessameud and Tayebi (2013) for the proof.

Since the desired orientation $Q_{d}$ should be tracked by the rotational dynamics and may be time-varying, for designing the torque input, the desired angular velocity and its time-derivative are needed. These signals can be obtained by

$$
\begin{aligned}
\omega_{d} & =\Xi(F) \dot{F}, \\
\dot{\omega}_{d} & =\dot{\Xi}(F, \dot{F}) \dot{F}+\Xi(F) \ddot{F},
\end{aligned}
$$

with

$$
\Xi(F)=\frac{1}{\gamma_{1}^{2} \gamma_{2}}\left(\begin{array}{ccc}
-\mu_{1} \mu_{2} & -\mu_{2}^{2}+\gamma_{1} \gamma_{2} & -\mu_{2} \gamma_{2} \\
\mu_{1}^{2}-\gamma_{1} \gamma_{2} & \mu_{1} \mu_{2} & -\mu_{1} \gamma_{2} \\
\mu_{2} \gamma_{1} & -\mu_{1} \gamma_{1} & 0
\end{array}\right)
$$

where $\gamma_{1}=\frac{\mathcal{T}}{m}, \quad \gamma_{2}=\gamma_{1}+\left(g-\mu_{3}\right)$ and $\dot{\Xi}(F, \dot{F})$ is the time-derivative of $\Xi(F)$.

\section{Designing the intermediary control}

In this section, we design the intermediary control for each vehicle with the second-order system described by (3). Before moving on, we state the following lemmas which will be used subsequently in this paper.

Lemma 4.1: Consider the second-order system

$$
\ddot{\theta}=-k_{p} \chi(\theta)-k_{d} \chi(\dot{\theta})+\varepsilon,
$$

where $\theta \in \mathbb{R}^{3}$ and $k_{p}, k_{d}>0$. If $\epsilon$ as a perturbation term is globally bounded and converges to zero, then $\theta$ and $\dot{\theta}$ are also globally bounded and converge to zero.

The proof is given in Abdessameud and Tayebi (2009).

Lemma 4.2: Consider the second-order system $\ddot{\xi}_{i}=u_{i}+$ $\varepsilon_{i}$ for $i=1, \ldots, n$ and $\epsilon_{i}$ is a bounded perturbation that converges to zero. We assume that the information exchange between agents switches over time and at each time instant contains a directed spanning tree. Assume that the following control input is

$$
\begin{aligned}
u_{i}= & \dot{v}_{d}-k\left(\dot{\xi}_{i}-v_{d}(t)\right) \\
& -\ell \sum_{j \in \mathcal{N}_{i}} a_{i j}^{\sigma}\left(\xi_{i}-\xi_{j}-\delta_{i j}\right),
\end{aligned}
$$

with gains $k$ and $\ell$ and dwell time $\tau_{0}$ satisfying

$$
\begin{aligned}
k & >0, \quad k^{2}>\frac{\ell \operatorname{Im}\left\{s_{i}\right\}}{\operatorname{Re}\left\{s_{i}\right\}}, \\
\tau_{0} & >\sup _{p \in \mathcal{P}}\left\{\frac{a^{p}}{\lambda^{p}}\right\},
\end{aligned}
$$

where $s_{i}, a^{p}$ and $\lambda^{p}$ defined in Appendix 2. Then, we will have

$$
\dot{\xi}_{i} \rightarrow v_{d}(t), \quad \xi_{i}-\xi_{j} \rightarrow \delta_{i j}
$$

where $\delta_{i j}=\delta_{i}-\delta_{j}$, and $\delta_{i}$ is the desired position of the vehicle $i$ from the centre of the formation and $a_{i j}$ is the entry of the adjacency matrix with $a_{i i}=0$ and $a_{i j}=1$ if vehicle $i$ can receive information from the jth one, otherwise $a_{i j}=0$.

The proof is given in Appendix 2.

Lemma 4.3: Consider the adaptation law

$$
\dot{\hat{b}}=\gamma^{b} \operatorname{proj}(\Upsilon, \hat{b})=\gamma^{b}\left(\Upsilon-\kappa \vartheta_{1} \vartheta_{2} \hat{b}\right), \quad \gamma^{b}>0(
$$

with

$$
\begin{gathered}
\kappa=\left(2\left(\epsilon^{2}+2 \epsilon B\right)^{2} B^{2}\right)^{-1}, \\
\vartheta_{1}=\left\{\begin{array}{cc}
\left(\hat{b}^{T} \hat{b}-B^{2}\right)^{2} & \hat{b}^{T} \hat{b}>B^{2} \\
0 & \text { otherwise }
\end{array},\right. \\
\vartheta_{2}=\hat{b}^{T} \Upsilon+\left(\left(\hat{b}^{T} \Upsilon\right)^{2}+\bar{\delta}^{2}\right)^{\frac{1}{2}},
\end{gathered}
$$

where $\varepsilon$ and $\bar{\delta}$ are arbitrary positive constants, $\hat{b}$ is the estimation of $b, \tilde{b}=b-\hat{b}, B>0$ is the bound on the estimation and $Y(t)$ is a known, continuously differentiable variable. Then, the following properties hold:

1) $\|\hat{b}\| \leq B+\epsilon, \quad \forall t>0$

2) $\tilde{b}^{T} \operatorname{proj}(\Upsilon, \hat{b}) \geq \tilde{b}^{T} \Upsilon$,

3) $\|\operatorname{proj}(\Upsilon, \hat{b})\| \leq\|\Upsilon\|[1+((B+\epsilon) / B)]^{2}+$ $\left((B+\epsilon) /\left(2 B^{2}\right)\right) \bar{\delta}$,

4) $\operatorname{proj}(\Upsilon, \hat{b}) \in \mathcal{C}^{1}$. 
The proof is given in Cai, DeQueiroz, and Dawson (2006).

Putting aside the underactuation error in (3), the rest can be regarded as a fully actuated second-order system with the control input $F_{i}$ possessing the following constraints

(1) Never touch the point $(0,0, g)^{T}$ such that condition (8) is always satisfied.

(2) Its first and second time-derivatives can be calculated explicitly in order to be used in designing torque input for the rotational dynamics.

Now we are ready to design the intermediary control $F_{i}$ for each aircraft such that the formation objective can be achieved while the above-mentioned constraints hold. The underactuation term $\tilde{F}_{i}$ can be viewed as a perturbation term which will be bounded if $F_{i}$ is bounded and will be vanished by convergence of the attitude of each aircraft to its desired attitude. To this end, we define the intermediary control $F_{i}$ and the following auxiliary systems for each vehicle as

$$
\begin{aligned}
F_{i} & =\dot{v}_{d}(t)-k_{\dot{\theta}_{i}} \chi\left(\dot{\theta}_{i}\right)-k_{\theta_{i}} \chi\left(\theta_{i}\right)-\hat{b}_{i}, \\
\ddot{\theta}_{i} & =-k_{\dot{\theta}_{i}} \chi\left(\dot{\theta}_{i}\right)-k_{\theta_{i}} \chi\left(\theta_{i}\right)-u_{i}, \\
\ddot{\alpha}_{i} & =u_{i}-\phi_{i}+\tilde{F}_{i}, \\
\ddot{\xi}_{i} & =\dot{v}_{d}(t)+\phi_{i}-\varphi_{i},
\end{aligned}
$$

with

$$
\begin{aligned}
u_{i} & =-k_{\dot{\alpha}_{i}} \dot{\alpha}_{i}-k_{\alpha_{i}} \alpha_{i}, \\
\phi_{i} & =-k\left(\dot{\xi}_{i}-v_{d}(t)\right)-\ell \sum_{j \in \mathcal{N}_{i}(t)} a_{i j}^{\sigma(t)} \xi_{i j},
\end{aligned}
$$

where $\xi_{i j}=\xi_{i}-\xi_{j}-\delta_{i j}$ and $\mathcal{N}_{i}$ is the set of neighbours of the $i$ th vehicle and the second-order auxiliary systems (22)-(24) can be initialised arbitrarily and $k_{\dot{\theta}_{i}}, k_{\theta_{i}}, k_{\dot{\theta}_{i}}, k_{\theta_{i}}$, $k_{\dot{\alpha}_{i}}, k, \ell$ are strictly positive scalar gains. We will treat $\xi_{i}$ and $\xi_{i}$, respectively, as a position and velocity of a virtual agent corresponding to the vehicle $i$ which is supposed to achieve the goal we expected from the actual vehicles. Then, by converging the position and velocity of each vehicle to those of its virtual vehicle, the desired coordination task will be acquired.

By defining a new variable,

$$
\zeta_{i}=p_{i}-\theta_{i}-\alpha_{i}-\xi_{i}
$$

Now we can have

$$
\ddot{\zeta}_{i}=\varphi_{i}+b_{i}-\hat{b}_{i}
$$

We design $\varphi_{i}$ such that $\zeta_{i}, \dot{\zeta}_{i} \rightarrow 0$ as

$$
\begin{gathered}
\varphi_{i}=-k_{\zeta_{i}} \zeta_{i}-\left(k_{\dot{\zeta}_{i}}+k_{b_{i}}\right) \dot{\zeta}_{i}, \\
\dot{\hat{b}}_{i}=\gamma_{i}^{b} \operatorname{proj}\left(\Upsilon_{i}^{b}, \hat{b}_{i}\right), \quad \Upsilon_{i}^{b}=\frac{k_{b_{i}} k_{\zeta_{i}}}{\gamma_{i}^{b}} \zeta_{i}+\left(\frac{k_{b_{i}} k_{\dot{\zeta}_{i}}}{\gamma_{i}^{b}}+1\right) \dot{\zeta}_{i},
\end{gathered}
$$

where $k_{\zeta_{i}}, k_{\dot{\zeta}_{i}}, k_{b_{i}}$ are positive scalar gains, $\operatorname{proj}($.$) is$ described in Lemma 4.3 and the initial value for estimator dynamics (30) should be taken such that $\left\|\hat{b}_{i}(0)\right\|<$ $B_{i}^{b}+\epsilon_{i}^{b}$ in which $B_{i}^{b}$ and $\epsilon_{i}^{b}$ are constants to be chosen for each aircraft to keep the estimation bounded by property $p 1$ of the projection operator. It should be noted that with the adaptation law $\dot{\hat{b}}_{i}=\Upsilon_{i}^{b}$, we can guarantee that the estimation of the translational disturbance moves towards its actual value. However, that does not ensure any boundedness for the estimation $\hat{b}_{i}$, and hence we use the smooth projection operator introduced in Cai et al. (2006) that enables us to design an adaptive control law which keeps the estimation within the priori bounded set, i.e. $\left\|b_{i}\right\|<B_{i}^{b}+\epsilon_{i}^{b}$, which consequently leads to a priori bounded intermediary control input $F_{i}$.

Remark 4.1: By means of the saturation function $\chi($. and projection operator defined in Lemma 4.3, the first constraint on $F_{i}$ that is mentioned earlier is satisfied. To satisfy the second condition, the auxiliary systems (22)(24) are introduced.

Remark 4.2: The idea behind using the third auxiliary system is to avoid differentiating a non-continuous function in the process of calculating $\ddot{F}_{i}$.

Since system (23) is perturbed by the term $\tilde{F}_{i}$, it is necessary for us to guarantee global boundedness of the perturbation term. This boundedness can be ensured by boundedness of designed intermediary control $F_{i}$. From Equation (21), we can determine the following bound on $F_{i}$ :

$$
F_{i} \leq\left\|\dot{v}_{d}\right\|+\epsilon_{i}^{b}+B_{i}^{b}+\sqrt{3}\left(k_{\theta_{i}}+k_{\dot{\theta}_{i}}\right) M
$$

Then, by Equation (6), we can say

$$
\mathcal{T}_{i} \leq m_{i}\left(g+\left\|\dot{v}_{d}\right\|+\epsilon_{i}^{b}+B_{i}^{b}+\sqrt{3}\left(k_{\theta_{i}}+k_{\dot{\theta}_{i}}\right) M\right)
$$

And finally, considering Equation (5), we can guarantee boundedness of the perturbation term $\tilde{F}_{i}$ by

$$
\tilde{F}_{i} \leq 2 \sqrt{2} \frac{\mathcal{T}_{i}}{m_{i}}\left\|\tilde{q}_{i}\right\|
$$




\section{Designing the torque input}

The unit quaternion error $\tilde{Q}_{i}=\left(\tilde{q}_{i}^{T}, \tilde{\eta}_{i}\right)^{T}$ is obtained by the quaternion multiplication as follows:

$$
\tilde{Q}_{i}=Q_{d_{i}}^{-1} \odot Q_{i},
$$

and the system error dynamics can be obtained by the

$$
\begin{aligned}
\dot{\tilde{Q}}_{i}= & \frac{1}{2}\left(\begin{array}{c}
\tilde{\eta}_{i} I_{3}+S\left(\tilde{q}_{i}\right), \\
-q_{i}^{T}
\end{array}\right) \tilde{\omega}_{i}, \\
J_{i} \dot{\tilde{\omega}}_{i}= & \Gamma_{i}-S\left(\tilde{\omega}_{i}\right) J_{i} \tilde{\omega}_{i}+S(\hat{z}) R\left(Q_{i}\right) d_{i} \\
& +J_{i}\left(S\left(\tilde{\omega}_{i}\right) R\left(\tilde{Q}_{i}\right) \omega_{d_{i}}-R\left(\tilde{Q}_{i}\right) \dot{\omega}_{d_{i}}\right),
\end{aligned}
$$

where

$$
\tilde{\omega}_{i}=\omega_{i}-R\left(\tilde{Q}_{i}\right) \omega_{d_{i}} .
$$

We define a new variable $\Omega_{i}$ as

$$
\Omega_{i}=\tilde{\omega}_{i}-\beta_{i}
$$

with

$$
\beta_{i}=-k_{\beta_{i}} \tilde{q}_{i}
$$

Then, by differentiating $\Omega_{i}$, we have

$$
\dot{\Omega}=\Gamma_{i}-H_{i}-J_{i} R\left(\tilde{Q}_{i}\right) \bar{\Pi}_{i} b_{i}+S(\hat{z}) R\left(Q_{i}\right) d_{i},
$$

where

$$
\begin{aligned}
H_{i}= & S\left(\omega_{i}\right) J_{i} \omega_{i}-J_{i} S\left(\tilde{\omega}_{i}\right) R\left(\tilde{Q}_{i}\right) \omega_{d_{i}} \\
& +J_{i} R\left(\tilde{Q}_{i}\right) \Pi_{i}+J_{i} \dot{\beta}_{i},
\end{aligned}
$$

with $\Pi_{i}$ and $\bar{\Pi}_{i}$ are defined in (59)-(60).

Now, we design the control torque and estimation of rotational disturbance for each vehicle as

$\Gamma_{i}=H_{i}-k_{Q_{i}} \tilde{q}_{i}-k_{\Omega_{i}} \Omega_{i}+J_{i} R\left(\tilde{Q}_{i}\right) \bar{\Pi}_{i} \hat{b}_{i}^{\prime}-S(\hat{z}) R\left(Q_{i}\right) \hat{d}_{i}$,

$$
\begin{aligned}
& \dot{\hat{d}}_{i}=\gamma_{i}^{d} \operatorname{proj}\left(\Upsilon_{i}^{d}, \hat{d}_{i}\right), \quad \Upsilon_{i}^{d}=-k_{i}^{d} R\left(Q_{i}\right)^{T} S(\hat{z}) \Omega_{i}, \\
& \dot{\hat{b}}_{i}^{\prime}=\gamma_{i}^{b^{\prime}} \operatorname{proj}\left(\Upsilon_{i}^{b^{\prime}}, \hat{b}_{i}^{\prime}\right), \quad \Upsilon_{i}^{b^{\prime}}=-k_{i}^{b^{\prime}} \bar{\Pi}_{i}^{T} R\left(Q_{i}\right)^{T} J_{i} \Omega_{i},
\end{aligned}
$$

where $\hat{b}_{i}^{\prime}$ is the second estimator of disturbance $b_{i}$.

The reason for defining the above structure for control torque is established through Lyapunov analysis fully discussed in the following section.

\section{Stability analysis}

In this section, the main result and the proof for the stability of the overall closed-loop system are presented. First, we state the main result in the following theorem.

Theorem 6.1: Consider the system in (1) and the intermediary control defined in (3)-(5) with the extraction algorithm described in Lemma 3.1. Assume the communication graph between vehicles switches by time with having a directed spanning tree at each time instant and suppose the dwell time $\tau_{0}$ satisfies condition (15). By designing the intermediary control $F_{i}$ as (21) with (25)-(30) and the torque input $\Gamma_{i}$ as given in (42) with $\beta_{i}$ given in (39) and taking gains such that condition (14) and the following condition is satisfied

$$
\left\|\dot{v}_{d}\right\|+B_{i}^{b}+\epsilon_{i}^{b}+\sqrt{3}\left(k_{\theta_{i}}+k_{\dot{\theta}_{i}}\right) M<g .
$$

Then, for each $i, j \in[1, \ldots, n]$, we have

$$
v_{i} \rightarrow v_{d}(t), \quad p_{i}-p_{j} \rightarrow \delta_{i}-\delta_{j} .
$$

\subsection{Proof}

Proof: Consider the Lyapunov function

$$
\begin{aligned}
V= & \sum_{i=1}^{n}\left(\frac{k_{\zeta_{i}}}{2} \zeta_{i}^{T} \zeta_{i}+\frac{1}{2} \dot{\zeta}_{i}^{T} \dot{\zeta}_{i}+\frac{1}{2 \gamma^{b}} \tilde{b}_{i}^{T} \tilde{b}_{i}\right) \\
& +\sum_{i=1}^{n}\left(\frac{1}{2} \Omega_{i} J_{i} \Omega_{i}+2 k_{Q_{i}}\left(1-\tilde{\eta}_{i}\right)\right. \\
& \left.+\frac{1}{2 \gamma_{i}^{d} k_{i}^{d}} \tilde{d}_{i}^{T} \tilde{d}_{i}+\frac{1}{2 \gamma_{i}^{b^{\prime}} k_{i}^{b^{\prime}}} \tilde{b}_{i}^{\prime T} \tilde{b}_{i}^{\prime}\right),
\end{aligned}
$$

with

$$
\tilde{b}_{i}=b_{i}-\hat{b}_{i}-k_{b_{i}} \dot{\zeta}_{i}, \quad \tilde{d}_{i}=d_{i}-\hat{d}_{i}, \quad \tilde{b}_{i}^{\prime}=b_{i}-\hat{b}_{i}^{\prime} .
$$

Differentiating the Lyapunov function along the trajectory of systems (28) and (40) and using the property $p 2$ of the projection operator stated in Lemma 4.3, we have

$$
\dot{V} \leq \sum_{i=1}^{n}-k_{\dot{\zeta}_{i}} \dot{\zeta}_{i}^{T} \dot{\zeta}_{i}-k_{\Omega_{i}} \Omega_{i}^{T} \Omega_{i}-k_{Q_{i}} k_{\beta_{i}} \tilde{q}_{i}^{T} \tilde{q}_{i}-\frac{k_{b_{i}}}{\gamma_{i}^{b}} \tilde{b}_{i}^{T} \tilde{b}_{i} .
$$

By satisfying condition (45), the extraction of the input thrust $\mathcal{T}_{i}$ and desired attitude $Q_{d_{i}}$ is always possible. From the Lyapunov function and its negative semi-definite time-derivative, respectively, given in (46) and (48), we 

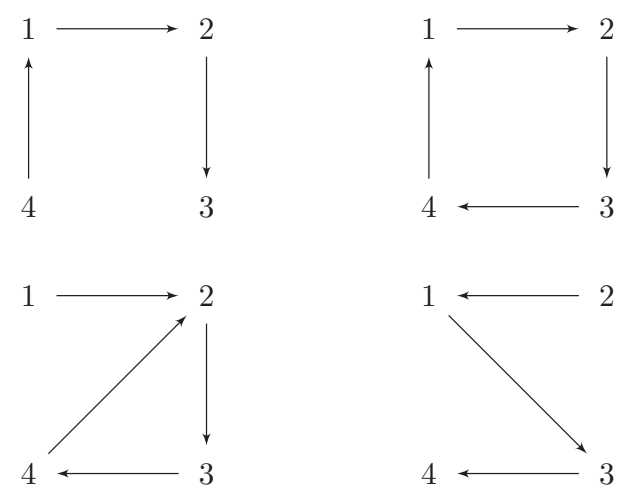

Figure 1. Four different directed topologies.

conclude that $\zeta_{i}, \dot{\zeta}_{i}, \tilde{b}^{\prime}, \tilde{b}_{i}, \Omega_{i},\left(1-\tilde{\eta}_{i}\right), \tilde{d}_{i}$ are globally bounded. From boundedness of $\zeta_{i}, \dot{\zeta}_{i}, \tilde{b}_{i}$ and Equations (28) and (29), we can conclude that $\ddot{\zeta}_{i}$ is bounded. Boundedness of $\tilde{b}_{i}$ and the property $p 3$ of the projection operator result in boundedness of $\bar{\Pi}_{i}, \Pi_{i}$ and $\dot{\tilde{b}}_{i}$. Since signals $\Omega_{i}, \tilde{q}_{i}, \bar{\Pi}_{i}, \tilde{b}_{i}$ and $\tilde{d}_{i}$ are bounded, one can conclude from Equations (40) and (42) that $\dot{\Omega}_{i}$ is also bounded. Since $\tilde{q}_{i}$ and $\tilde{\Omega}_{i}$ are bounded, $\tilde{\omega}_{i}$ is bounded and consequently $\dot{\tilde{q}}_{i}$ is bounded. Hence, we see that $\ddot{V}$ is bounded which follows with uniform continuity of $\dot{V}$. Invoking Barbalat's lemma leads to $\dot{V} \rightarrow 0$ and as a result $\dot{\zeta}_{i}, \Omega_{i}, \tilde{q}_{i}$ converge asymptotically to zero and $\hat{b}_{i} \rightarrow b_{i}$. Boundedness and convergence of $\dot{\zeta}_{i}$ to zero follows with the bounded $\varphi_{i}$ that converges to zero. Using Lemma 4.2 , we can prove $\xi_{i}-\xi_{j}$ $\rightarrow \delta_{i j}, \dot{\xi} \rightarrow \dot{v}_{d}$. From this and Equation (26), we can say $\phi_{i}$ is bounded and converges to zero. The term $\tilde{F}_{i}$ also
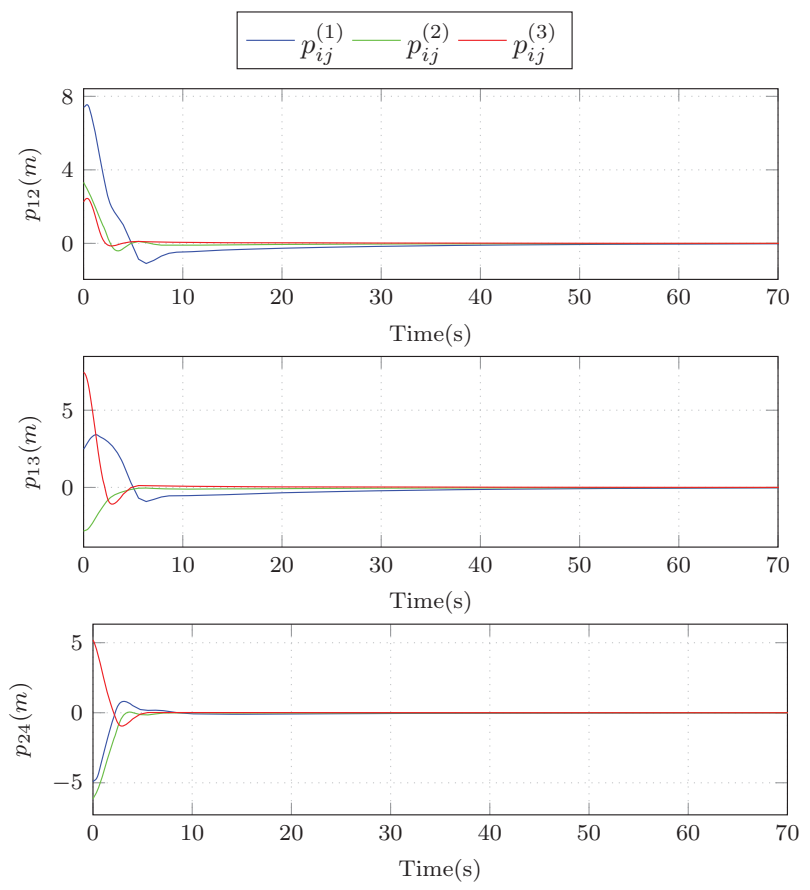

Figure 3. Formation error $p_{i j}=p_{i}-p_{j}-\underline{\delta}_{i j}=\left(p_{i j}^{(1)}, p_{i j}^{(2)}\right.$, $\left.p_{i j}^{(3)}\right)^{T}$.

is bounded by (31) and converges to zero with converging $\tilde{q}_{i}$. Regarding $\phi_{i}+\tilde{F}_{i}$ as a vanishing perturbation for system (23), it can be easily proved that $\alpha_{i}$ and $\dot{\alpha}_{i}$ are bounded and $\alpha_{i}, \dot{\alpha}_{i} \rightarrow 0$. Boundedness and convergence of $\theta_{i}, \dot{\theta}_{i}$ to zero can also be easily shown using Lemma 3.1. Therefore, from (27), we can see $p_{i} \rightarrow \xi_{i}$ and $v_{i} \rightarrow \dot{\xi}_{i}$. Finally, we can say that $v_{i} \rightarrow v_{d}(t)$ and $\left(p_{i}-p_{j}-\delta_{i j}\right) \rightarrow$ 0 .

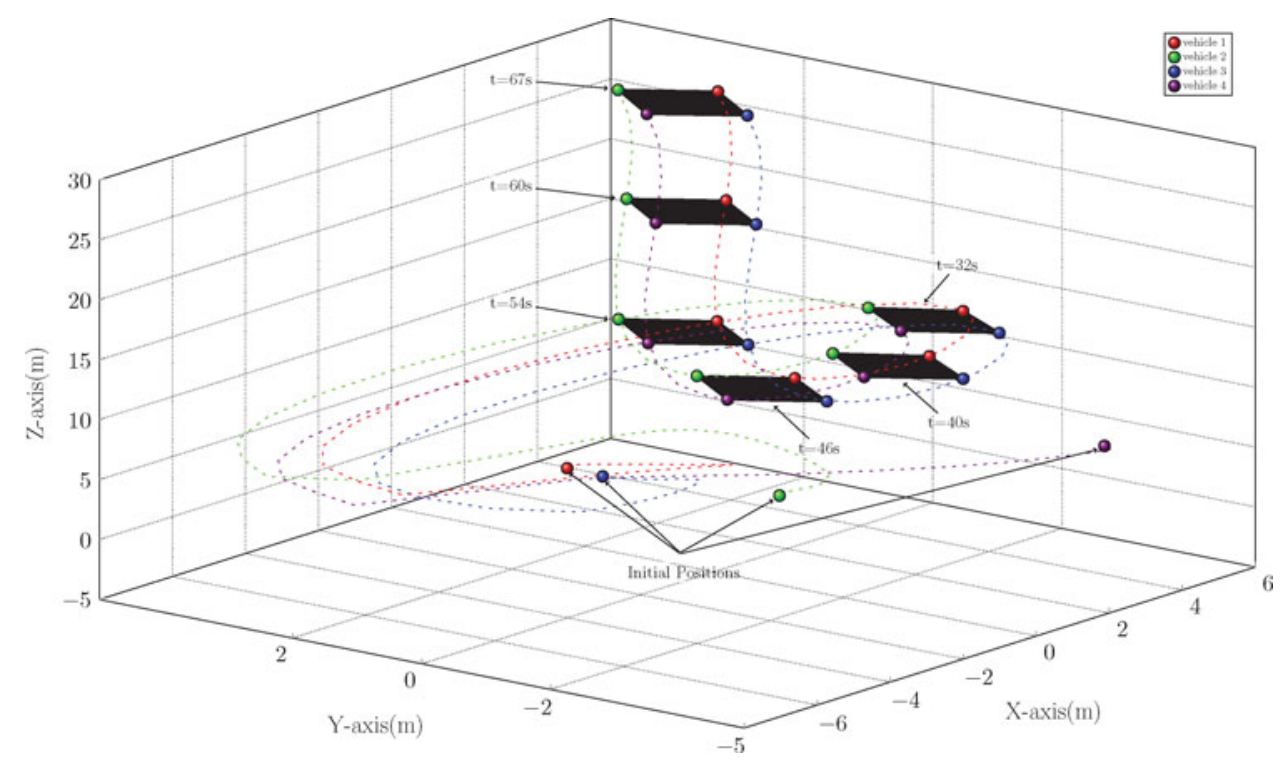

Figure 2. Position of vehicles. 


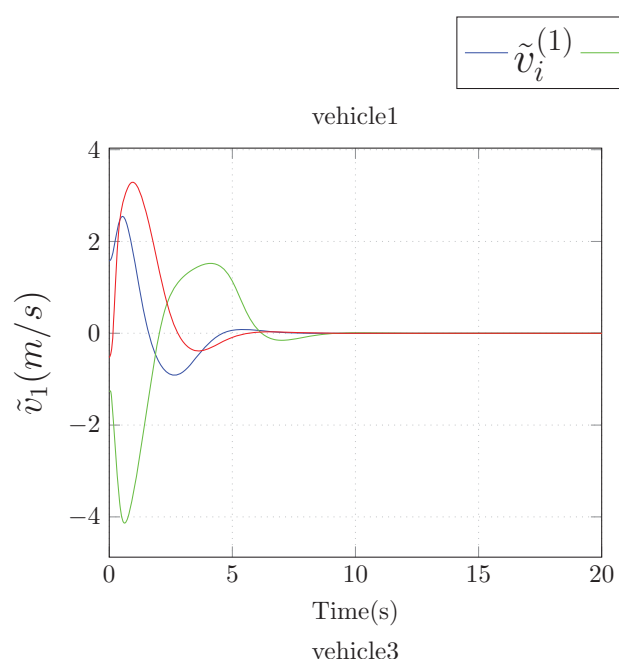

$\tilde{v}_{i}^{(2)}-\tilde{v}_{i}^{(3)}$
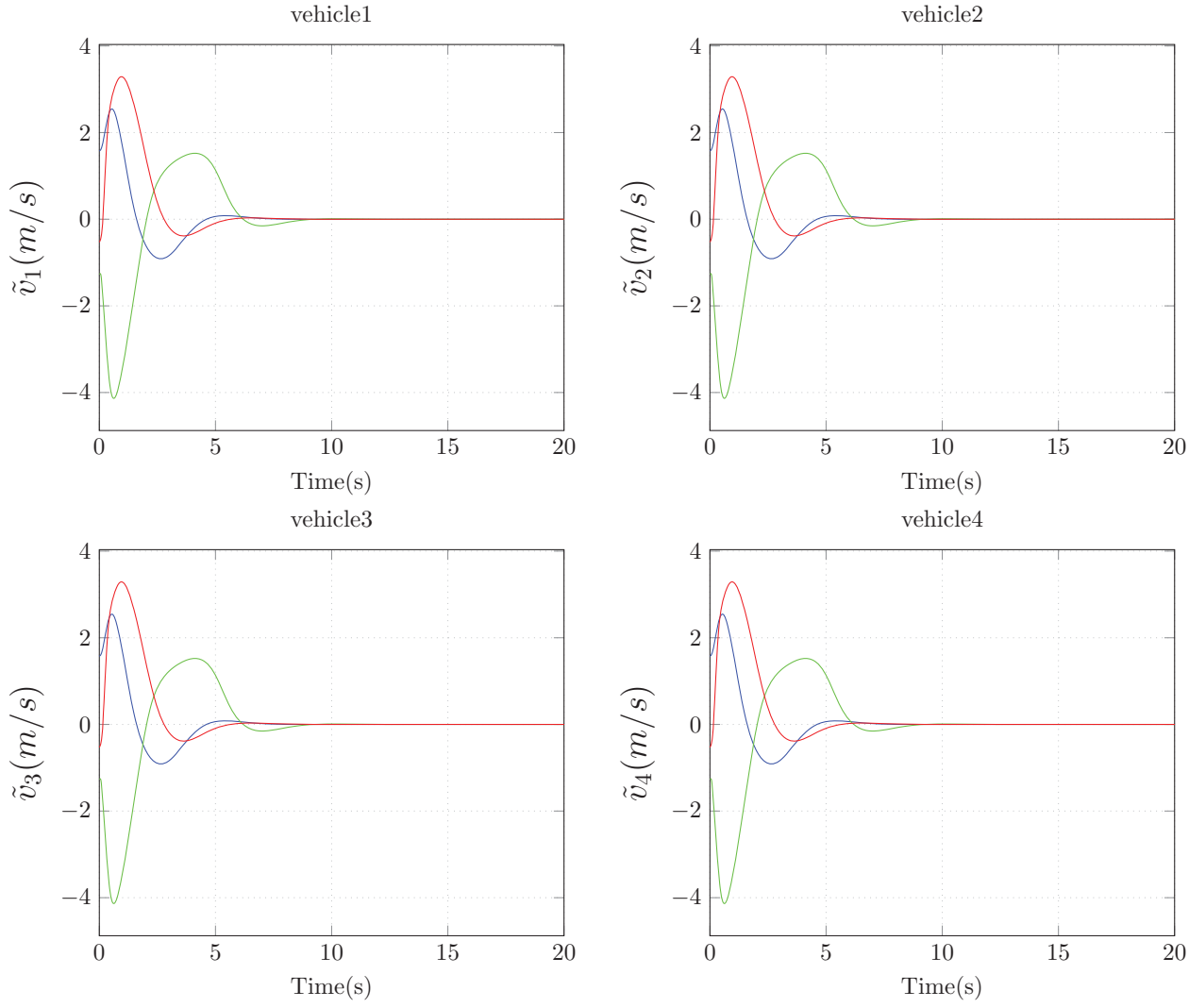

Figure 4. Linear velocity error $\tilde{v}_{i}=\left(\tilde{v}_{i}^{(1)}, \tilde{v}_{i}^{(2)}, \tilde{v}_{i}^{(3)}\right)^{T}$.

\section{Simulation results}

Table 1. Parameters and gains used in simulations.

In this section, numerical simulations are given to illustrate the obtained theoretical results. A scenario with $n=4$ vehicles is considered with $J_{i}=\operatorname{diag}(0.3,0.4$, $0.25) \mathrm{kg} \mathrm{m}^{2}, m_{i}=5 \mathrm{~kg}, Q_{i}(0)=(0,0,0,1)$ and $\omega_{i}(0)=(0,0,0)$ for $i=1, \ldots, 4$. The information graph between agents switches randomly between four weakly connected graphs illustrated in Figure 1 at every $0.1 \mathrm{~s}$. The weights on each edge are set to be 1 . The initial positions and linear velocities are randomly selected, respectively, in $[-5,5]$ and $[-1,1]$. The initial values of the all auxiliary systems and the estimators are set to be zero. The offset vectors are selected as $\delta_{1}=(1,0,0), \delta_{2}=(-1,0$, $0), \delta_{3}=(0,1,0), \delta_{4}=(0,-1,0)$. The other gains and parameters are shown in Table 1.

To test the validity of the estimation adaptive laws, the translational disturbances and torque disturbances are chosen to be $b_{i}=(0.5,0.3,0.4)^{T} \mathrm{~m} / \mathrm{s}^{2}, d_{i}=(0.3,0.4$, $0.8)^{T}$ N.m. The desired linear velocity is of the form $v_{d}(t)$ $=((-2.5 / \pi) \cos (t /(2 \pi)),(1.25 / \pi) \cos (t /(3 \pi)), 0.4)$ and $\tanh ($.$) is selected as an example of the smooth saturation$ function $\varrho($.$) with M=1$.

The results are shown in Figures 2-8. The positions of the vehicles are shown in Figure 2 where vehicles are the vertices of the rectangles. As we can see, all vehicles gradually converge to a rectangle shape which is determined by the offset vectors and while keeping this formation, track the linear velocity $v_{d}(t)$. The results can also be seen in Figures 3 and 4 which, respectively, illustrate the relative positions error $p_{i j}=p_{i}-p_{j}-\delta_{i j}$ and the velocity error $\tilde{v}_{i}=v_{i}-v_{d}(t)$. The estimations of the translational disturbances, $\hat{b}_{i}$, are shown in Figure 5. Norms of the vector part of the attitude errors $\left\|\tilde{q}_{i}\right\|$ are given in Figure 6. Figures 7 and 8 show the input thrust $T_{i}$ and the norm of the torque input $\left\|\Gamma_{i}\right\|$ for each vehicle. 


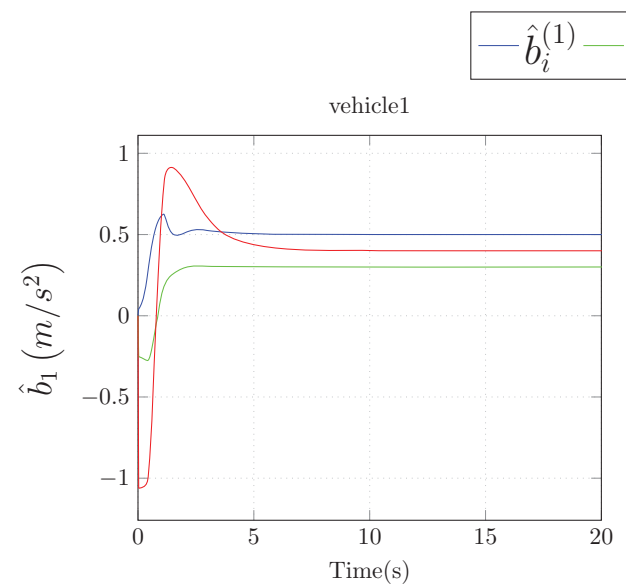

$\hat{b}_{i}^{(2)}-\hat{b}_{i}^{(3)}$
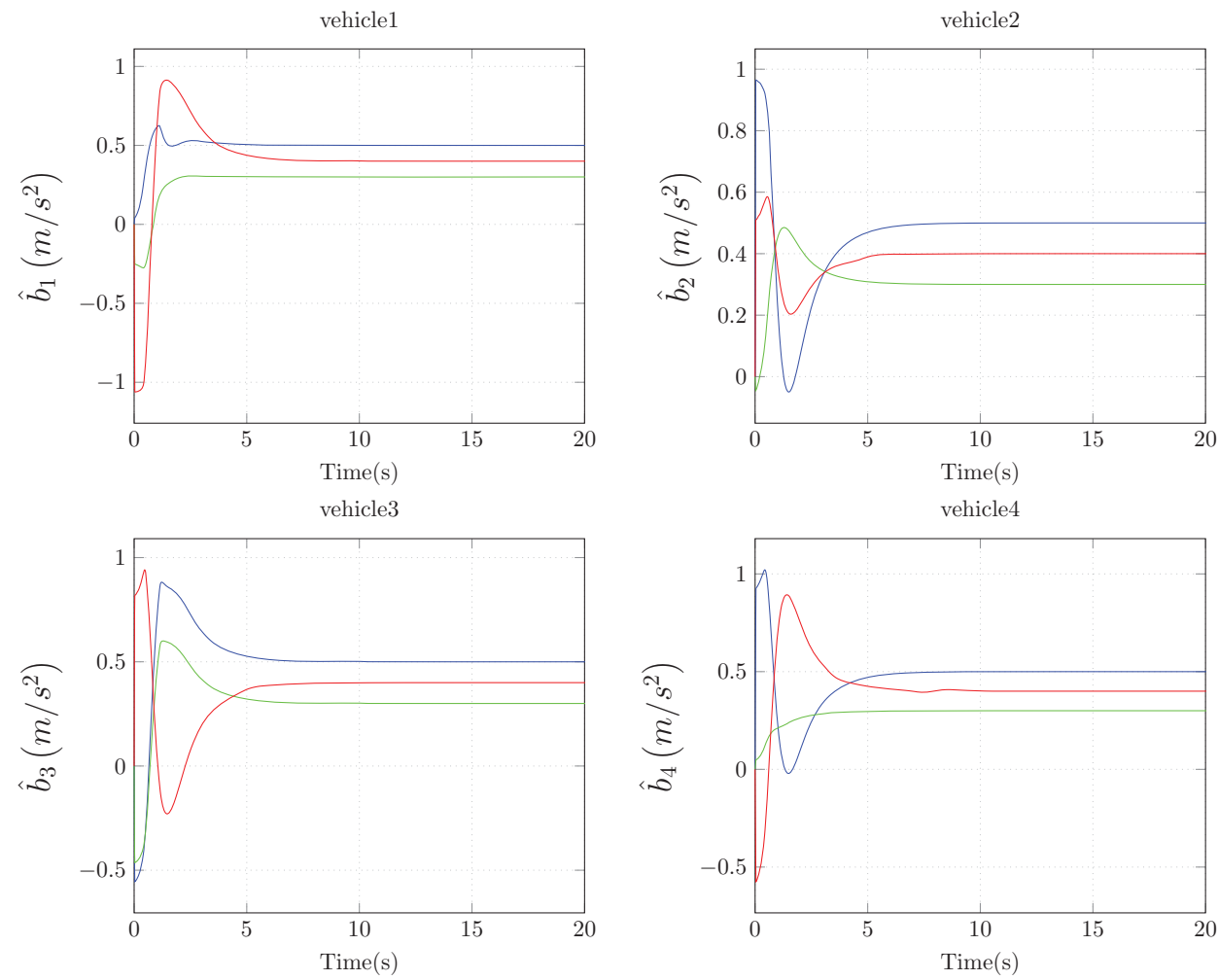

Figure 5. Estimation of translational disturbance $\hat{b}_{i}=\left(\hat{b}_{i}^{(1)}, \hat{b}_{i}^{(2)}, \hat{b}_{i}^{(3)}\right)^{T}$.

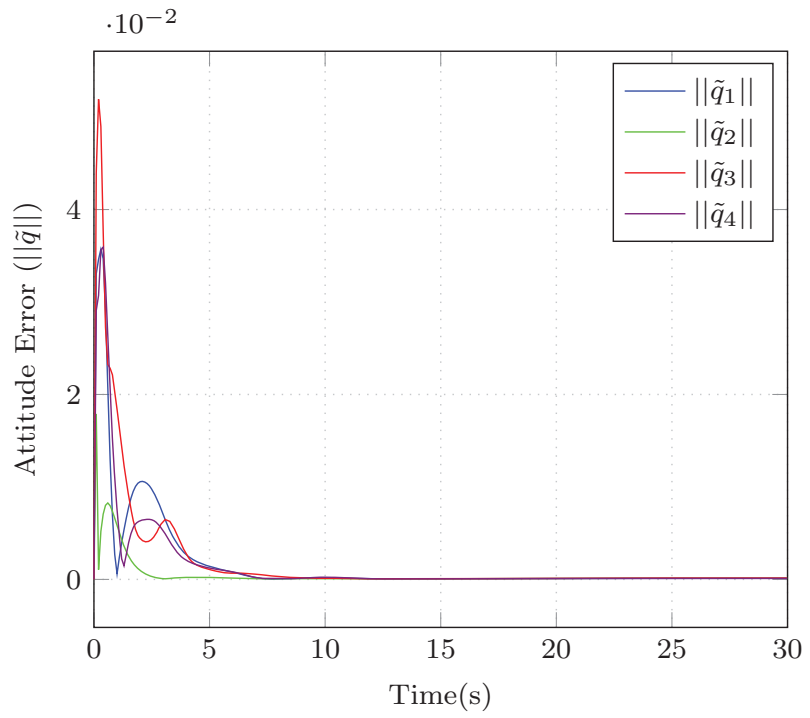

Figure 6. Norm of attitude error $\left\|\tilde{q}_{i}\right\|$.

\section{Conclusion}

The distributed coordination control problem for a team of multiple VTOL UAVs has been studied in this paper. Assuming that communication interaction

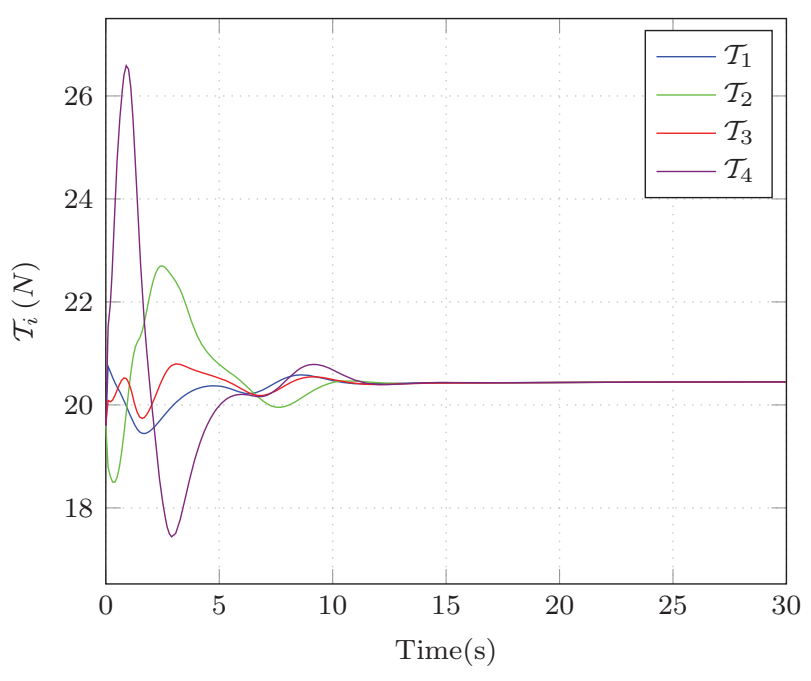

Figure 7. Magnitude input thrust $\mathcal{T}_{i}$.

topology evolves between graphs with spanning tree and by setting a lower bound on dwell time, we proved that the group of multiple VTOL UAVs globally achieves formation. Designing auxiliary systems and introducing virtual agents for each aircraft enabled us to cope with the underactuation nature of this type of systems. Furthermore, to 


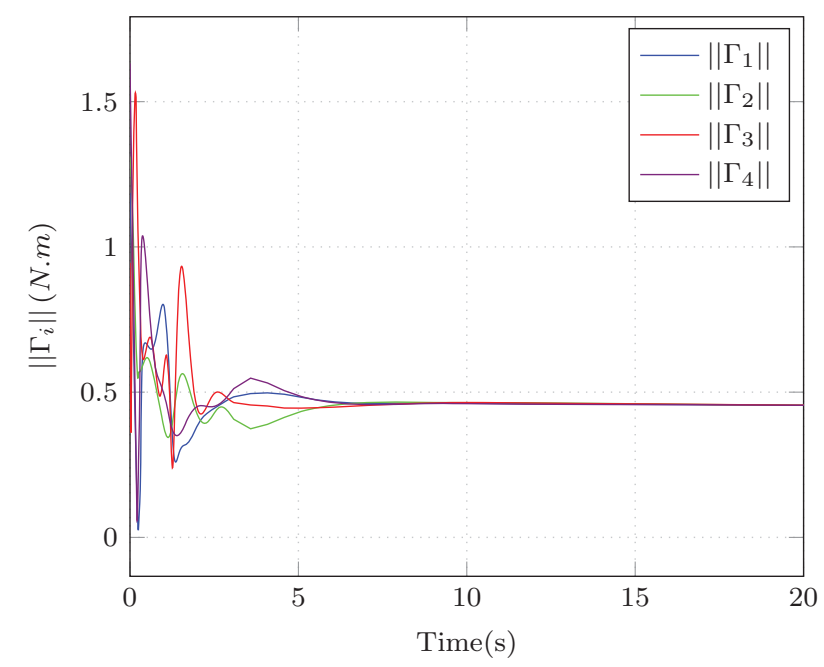

Figure 8. Norm of torque input $\left\|\Gamma_{i}\right\|$.

compensate for the adverse effect of the constant disturbances, we make use of smooth adaptive control laws. Finally, the simulation results were given to support the theoretical analysis.

\section{Disclosure statement}

No potential conflict of interest was reported by the authors.

\section{References}

Abdessameud, A., \& Tayebi, A. (2009, December). Formation control of VTOL-UAVs. In Proceedings of the 48h IEEE Conference on Decision and Control (CDC) Held Jointly with 2009 28th Chinese Control Conference. (pp. 3454-3459). Shanghai, China: IEEE. Retrieved from http://ieeexplore.ieee.org/lpdocs/epic03/wrapper.htm? arnumber $=5400941$

Abdessameud, A., \& Tayebi, A. (2011, November). Formation control of VTOL unmanned aerial vehicles with communication delays. Automatica, 47(11), 2383-2394. Retrieved from http://linkinghub.elsevier.com/retrieve/pii/ S0005109811004158

Abdessameud, A., \& Tayebi, A. (2013). Motion coordination for VTOL unmanned aerial vehicles. London: Springer. Retrieved from http://link.springer.com/10.1007/978-14471-5094-7

Abdessameud, A., Tayebi, A., \& Polushin, I.G. (2012, Sep). Attitude synchronization of multiple rigid bodies with communication delays. IEEE Transactions on Automatic Control, 57(9), 2405-2411. Retrieved from http://ieeexplore. ieee.org/lpdocs/epic03/wrapper.htm?arnumber $=6155071$

Atrianfar, H., \& Haeri, M. (2015, Dec.). Synchronous Consensus of Double-Integrator Continuous-time Multi-Agent Systems with Switching Topologies and Time-Varying Delays, In 54th IEEE Conference on Desicion and Control (CDC) (pp. 2205-2210). Osaka, Japan. Retrieved from http://ieeexplore.ieee.org/abstract/document/7402534/? section $=$ abstract
Atrianfar, H., Haeri, M. (2012, Oct.). On properties of a particular class of directed graphs used in stability analysis of flocking algorithms. 2012 IEEE International Conference on Control Applications (CCA) (pp. 605-608). Dubrovnik, Croatia. Retrieved from http://ieeexplore.ieee.org/document/6402445/

Atrianfar, H., Haeri, M. (2013, Oct.). Theoretical analysis of flocking algorithms in networks of second order dynamic agents with switching topologies. Journal of Dynamic Systems, Measurement and Control, 136, 1, 011013, Retrieved from http://dynamicsystems.asmedigitalcollection.asme.org/ article.aspx?articleid $=1753981$

Cabecinhas, D., Cunha, R., \& Silvestre, C. (2014, may). A nonlinear quadrotor trajectory tracking controller with disturbance rejection. In Cont. eng. pract. 26, pp. 1-10. Elsevier BV. Retrieved from http:// linkinghub.elsevier.com/retrieve/pii/S0967066114000343

Cai, Z., DeQueiroz, M., \& Dawson, D. (2006, January). A sufficiently smooth projection operator. IEEE Transactions on Automatic Control, 51(1), 135-139. Retrieved from http://ieeexplore.ieee.org/lpdocs/epic03/wrapper.htm? arnumber $=1576865$

Diebel, J. (2006). Representing attitude: Euler angles, unit quaternions, and rotation vectors. Matrix, 58, 1-35. Retrieved from ftp://sbai2009.ene.unb.br/Projects/ GPS-IMU/George/arquivos/Bibliografia/79.pdf

Erdong, J., \& Zhaowei, S. (2009, Mar). Robust attitude synchronisation controllers design for spacecraft formation. IET Control Theory \& Applications, 3(3), 325-339. Retrieved from http://digital-library.theiet.org/content/journals/ 10.1049/iet-cta_20070240

Hu, J., \& Zhang, $\bar{H}$. (2015, Mar). Output feedback control for rigid-body attitude with constant disturbances. International Journal of Control, 88(3), 602-612. Retrieved from http://www.tandfonline.com/doi/abs/10.1080/00207179. 2014.971342

Hua, M.-D., Hamel, T., Morin, P., \& Samson, C. (2013, February). Introduction to feedback control of underactuated VTOL vehicles. IEEE Control Systems Magazine, 33(1), 6175.

Khalil, H.K. (2002). Nonlinear systems (3rd ed.). Retrieved from http://cdsweb.cern.ch/record/1173048

Kopfstedt, T., Mukai, M., Fujita, M., \& Ament, C. (2008, Jul). Control of formations of UAVs for surveillance and reconnaissance missions. IFAC Proceeding, 41(2), 5161-5166. Retrieved from http://linkinghub.elsevier.com/retrieve/pii/ S1474667016397622

Lee, D. (2012, July). Distributed backstepping control of multiple thrust-propelled vehicles on a balanced graph. Automatica, 48(11), 2971-2977. Retrieved from http://linkinghub.elsevier.com/retrieve/ pii/S0005109812004062

Lin, P., \& Jia, Y. (2010, March). Consensus of a class of secondorder multi-agent systems with time-delay and jointlyconnected topologies. IEEE Transactions on Automatic Control, 55(3), 778-784. Retrieved from http://ieeexplore. ieee.org/lpdocs/epic03/wrapper.htm?arnumber $=5406004$

Morse, A. (1996). Supervisory control of families of linear set-point controllers - Part I. Exact matching. IEEE Transactions on Automatic Control, 41(10), 1413-1431. Retrieved from 
http://ieeexplore.ieee.org/lpdocs/epic03/wrapper.htm? arnumber $=539424$

Nigam, N., Bieniawski, S., Kroo, I., \& Vian, J. (2012, Sep). Control of multiple UAVs for persistent surveillance: Algorithm and flight test results. IEEE Transactions on Control Systems Technology, 20(5), 1236-1251. Retrieved from http://ieeexplore.ieee.org/lpdocs/epic03/wrapper.htm? arnumber $=6045299$

Olfati-Saber, R., \& Murray, R. (2004, September). Consensus problems in networks of agents with switching topology and time-delays. IEEE Transactions on Automatic Control, 49(9), 1520-1533. Retrieved from http://ieeexplore.ieee.org/lpdocs/epic03/wrapper.htm? arnumber $=1333204$

Pack, D., DeLima, P., Toussaint, G., \& York, G. (2009, Aug). Cooperative control of UAVs for localization of intermittently emitting mobile targets. IEEE Transactions on Systems, Man, and Cybernetics, Part B, 39(4), 959-970. Retrieved from http://ieeexplore.ieee.org/lpdocs/epic03/ wrapper.htm?arnumber $=4967999$

Pflimlin, J., Soueres, P., \& Hamel, T. (2004). Hovering flight stabilization in wind gusts for ducted fan UAV. In 2004 43 rd ieee. conf. decis. control (ieee cat. no.04ch37601) (Vol. 4, pp. 3491-3496). IEEE. Retrieved from http://ieeexplore.ieee.org/lpdocs/epic03/wrapper.htm? arnumber $=1429251$

Pflimlin, J.M., Soueres, P., \& Hamel, T. (2007, May). Position control of a ducted fan VTOL UAV in crosswind. International Journal of Control, 80(5), 666-683. Retrieved from http://www.tandfonline.com/doi/abs/10.1080/ 0207170601045034

Qin, J., Gao, H., \& Zheng, W.X. (2011, Jun). Second-order consensus for multi-agent systems with switching topology and communication delay. Systems \& Control Letters, 60(6), 390-397. Retrieved from http://linkinghub. elsevier.com/retrieve/pii/S0167691111000600

Ren, W. (2006). Distributed attitude consensus among multiple networked spacecraft. 2006 American control conference (Vol. 1, pp. 6). MN, USA . IEEE. Retrieved from http://ieeexplore.

ieee.org/lpdocs/epic03/wrapper.htm?arnumber $=1656474$

Ren, W., \& Beard, R.W. (2008). Distributed consensus in multi-vehicle cooperative control. InVol. 53 of Communications and control engineering. London: Springer London. Retrieved from http://link.springer.com/10.1007/9781-84800-015-5

Roberts, A., \& Tayebi, A. (2011, Feb). Adaptive position tracking of VTOL UAVs. IEEE Transactions on Robotics, 27(1), 129-142. Retrieved from http://ieeexplore.ieee.org/lpdocs/ epic03/wrapper.htm?arnumber $=5674091$

Roza, A., \& Maggiore, M. (2014, Sept). A class of position controllers for underactuated VTOL vehicles. IEEE Transactions on Automatic Control, 59(9), 2580-2585. Retrieved from http://ieeexplore.ieee.org/ stamp/stamp.jsp?tp $=$ \&arnumber $=6748916$ \&isnumber $=$ 6880868

Shuster, M.D. (1993). A survey of attitude representations. Navigation, 8(9), 439-517.

Tayebi, A. (2008, July). Unit quaternion-based output feedback for the attitude tracking problem. IEEE Transactions on Automatic Control, 53(6), 1516-1520. Retrieved from http://ieeexplore.ieee.org/lpdocs/epic03/wrapper.htm? arnumber $=4601498$

Zhang, Y., \& Tian, Y.P. (2009, May). Consentability and protocol design of multi-agent systems with stochastic switching topology. Automatica, 45(5), 1195-1201. Retrieved from http://linkinghub.elsevier.com/retrieve/pii/ S0005109808005451

Zheng, Y., \& Wang, L. (2012, December). Distributed consensus of heterogeneous multi-agent systems with fixed and switching topologies. International Journal of Control, 85(12), 1967-1976. Retrieved from http://www.tandfonline.com/doi/abs/ 10.1080/00207179.2012.713986

\section{Appendices Appendix 1. Calculating the known and unknown part of $\dot{\omega}_{d}$}

In this section, we calculate the time-derivative of the desired extracted angular velocity, $\dot{\omega}_{i}$. As $\ddot{\zeta}_{i}$ given in (28) contains the unknown signal $b_{i}$, in the process of obtaining $\dot{\omega}_{d_{i}}$, we confront with some unknown terms. Here, we group results into two parts, with known signals and unknown signals. For notational convenience, we obviate the subscript $i$. First, we calculate the second time-derivative of the estimation, $\ddot{\hat{b}}$. Before doing this, we need to differentiate some signals as follows:

$$
\begin{gathered}
\dot{\Upsilon}^{b}=\frac{k^{b} k_{\zeta}}{\gamma^{b}} \dot{\zeta}+\left(\frac{k_{b} k_{\dot{\zeta}}}{\gamma^{b}}+1\right)(\varphi-\hat{b}+b), \\
\dot{\vartheta}_{1}=\left\{\begin{array}{cc}
4\left(\hat{b}^{T} \hat{b}-B^{2}\right) \dot{\hat{b}}^{T} & \hat{b} \hat{b}^{T} \hat{b}>B^{2} \\
0 & \text { otherwise }
\end{array}\right. \\
\dot{\vartheta}_{2}=\Pi^{1}+\bar{\Pi}^{1} \hat{b}^{T} b,
\end{gathered}
$$

with

$$
\begin{gathered}
\Pi^{1}=\left(1+\hat{b}^{T} \Upsilon^{b}\left(\left(\hat{b}^{T} \Upsilon^{b}\right)^{2}+\bar{\delta}^{2}\right)^{-\frac{1}{2}}\right) \times \\
\left.\left(\dot{\hat{b}}^{1} \Upsilon^{b}+\frac{k^{b} k_{\zeta}}{\gamma^{b}} \hat{b}^{T} \dot{\zeta}+\left(\frac{k_{b} k_{\dot{\zeta}}}{\gamma^{b}}+1\right) \hat{b}^{T}(\varphi-\hat{b})\right)\right), \\
\bar{\Pi}^{1}=\left(1+\hat{b}^{T} \Upsilon^{b}\left(\left(\hat{b}^{T} \Upsilon^{b}\right)^{2}+\bar{\delta}^{2}\right)^{-\frac{1}{2}}\right)\left(\frac{k_{b} k_{\dot{\zeta}}}{\gamma^{b}}+1\right),
\end{gathered}
$$

where $\kappa, \vartheta_{1}$ and $\vartheta_{2}$ are given in Lemma 4.3.

Now according to (30), we can calculate $\ddot{\hat{b}}$ by

$$
\begin{aligned}
\ddot{\hat{b}} & =\gamma^{b}\left(\dot{\Upsilon}^{b}-\kappa \dot{\vartheta}_{1} \vartheta_{2} \hat{b}-\kappa \vartheta_{1} \dot{\vartheta}_{2} \hat{b}-\kappa \vartheta_{1} \vartheta_{2} \dot{\hat{b}}\right) \\
& =\Pi^{2}+\bar{\Pi}^{2} b,
\end{aligned}
$$

where

$$
\Pi^{2}=k^{b} k_{\zeta} \dot{\zeta}+\left(k_{b} k_{\dot{\zeta}}+\gamma^{b}\right)(\varphi-\hat{b})
$$




$$
\begin{array}{r}
-\gamma^{b} \kappa \dot{\vartheta}_{1} \vartheta_{2} \hat{b}-\gamma^{b} \kappa \vartheta_{1} \vartheta_{2} \dot{\hat{b}}-\gamma^{b} \kappa \vartheta_{1} \Pi \hat{b}, \\
\bar{\Pi}^{2}=\gamma^{b}\left(\kappa \vartheta_{1} \bar{\Pi}^{1} \hat{b} \hat{b}^{T}+\left(\frac{k_{b} k_{\dot{\zeta}}}{\gamma^{b}}+1\right) I_{3}\right) .
\end{array}
$$

From the equation given by (10), the second timederivative of the intermediary control can be written as $\ddot{F}=\Pi^{3}+\bar{\Pi}^{3} b$, where $\Pi^{3}$ and $\bar{\Pi}^{3}$ are given by

$$
\begin{aligned}
& \Pi^{3}=v_{d}^{(3)}(t)-k_{\theta} \dot{h}(\theta, \dot{\theta}) \dot{\theta}-\left(k_{\theta} h(\theta)-k_{\dot{\theta}} h(\dot{\theta})\right) \ddot{\theta} \\
& -k_{\dot{\theta}} h(\dot{\theta})\left(-k_{\theta} h(\theta) \dot{\theta}-k_{\dot{\theta}} \ddot{\theta}+k_{\alpha} \dot{\alpha}+k_{\dot{\alpha}} \ddot{\alpha}\right)-\Pi^{2} \\
& \bar{\Pi}^{3}=-\bar{\Pi}^{2} .
\end{aligned}
$$

where $h(x)=\operatorname{diag}\left(\frac{d \varrho\left(x_{1}\right)}{d x_{1}}, \frac{d \varrho\left(x_{2}\right)}{d x_{2}}, \frac{d \varrho\left(x_{3}\right)}{d x_{3}}\right)$ and $\dot{h}(x, \dot{x})$ is the time-derivative of $h(x)$.

At the end, from Equation (10) we have

$$
\dot{\omega}_{d}=\Pi+\bar{\Pi} b,
$$

with

$$
\begin{aligned}
\Pi & =\dot{\Xi}(F, \dot{F}) \dot{F}+\Xi(F) \Pi^{3}, \\
\bar{\Pi} & =\Xi(F) \bar{\Pi}^{3} b .
\end{aligned}
$$

\section{Appendix 2. Proof of Lemma 4.2}

In this section, we present the proof of Lemma 4.2. Let define the following variables:

$$
\begin{gathered}
\bar{\xi}_{i}=\xi_{i}-\delta_{i}, \\
Y=\left(\begin{array}{ll}
y_{1}^{T} & y_{2}^{T}
\end{array}\right)^{T},
\end{gathered}
$$

with

$$
\begin{aligned}
& y_{1}=\left(\left(\bar{\xi}_{1}-\bar{\xi}_{2}\right)^{T},\left(\bar{\xi}_{1}-\bar{\xi}_{3}\right)^{T}, \ldots\left(\bar{\xi}_{1}-\bar{\xi}_{n}\right)^{T}\right)^{T}, \\
& y_{2}=\left(\left(\dot{\xi}_{1}-v_{d}\right)^{T},\left(\dot{\xi}_{2}-v_{d}\right)^{T}, \ldots,\left(\dot{\xi}_{n}-v_{d}\right)^{T}\right)^{T} .
\end{aligned}
$$

Regarding the system given Lemma 4.2 and the input given in (13), we can write

$$
\dot{Y}=\left(C^{\sigma} \otimes I_{3}\right) Y+\bar{\varepsilon},
$$

where

$$
\mathrm{E}=\left(1_{n-1}-I_{n-1}\right) \in \mathbb{R}^{(n-1) \times n},
$$

$$
\begin{gathered}
\mathrm{F}=\left(\begin{array}{c}
0_{n-1}^{T} \\
-I_{n-1}
\end{array}\right) \in \mathbb{R}^{n \times(n-1)}, \\
C^{\sigma}=\left(\begin{array}{cc}
0_{(n-1 \times n-1)} & \mathrm{E} \\
-\ell \mathcal{L}^{\sigma} \mathrm{F} & -k I_{n}
\end{array}\right)_{(2 n-1) \times(2 n-1)}, \\
\bar{\varepsilon}=\left(\begin{array}{lll}
0_{1 \times 3(n-1)} & \varepsilon_{1}^{T} \ldots \varepsilon_{n}^{T}
\end{array}\right)^{T} .
\end{gathered}
$$

With the help of Lemma 1 in Zhang and Tian (2009), one can prove that the real part of all eigenvalues of the matrix $\left(\mathrm{E} \mathcal{L}^{\sigma} \mathrm{F}\right)$ is positive if and only if the topology graph contains a spanning tree.

Now we want to prove that all eigenvalues of $C^{\sigma}$ have negative real parts. The eigenvalues of $C^{\sigma}, \bar{\lambda}_{i}$, are the roots of the following determinant:

$$
\begin{aligned}
& \operatorname{det}\left(\begin{array}{l}
\bar{\lambda} I_{n-1} \\
\ell \mathcal{L}^{\sigma} \mathrm{F}(\bar{\lambda}+k) I_{n}
\end{array}\right)= \\
& \operatorname{det}\left(\bar{\lambda} I_{n-1}+\frac{\ell}{\bar{\lambda}+k} \mathrm{E} \mathcal{L}^{\sigma} \mathrm{F}\right) \operatorname{det}\left((\bar{\lambda}+k) I_{n}\right)=0,
\end{aligned}
$$

where we have exploited the fact that $\operatorname{det}\left(\begin{array}{ll}A & B \\ C & D\end{array}\right)=$ $\operatorname{det}\left(A-B D^{-1} C\right) \operatorname{det}(D)$. We can say that $\bar{\lambda}_{1}=-k$ and $2 n-2$ other eigenvalues of the $C^{\sigma}$ are the solutions of $n-$ 1 second-order polynomial equations $\bar{\lambda}^{2}+k \bar{\lambda}+\ell s_{i}=0$ which give two solutions for each $s_{i}$, for $i=1, \ldots, n-$ 1 , where $s_{i}$ is the $i$ th eigenvalue of the matrix $\left(\mathrm{E} \mathcal{L}^{\sigma} \mathrm{F}\right)$. By applying the Routh criterion, the real part of the secondorder polynomial equations will be negative if $k>0$ and $k^{2}>\frac{\ell\left(\operatorname{Im}\left\{s_{i}\right\}\right)^{2}}{\operatorname{Re}\left\{s_{i}\right\}}$.

Lemma B1: Let $C^{p}: p \in \mathcal{P}$ be a closed, bounded set of real, $n \times n$ matrices. Suppose that for each $p \in \mathcal{P}, C^{p}$ is stable and let $a^{p}$ and $\lambda^{p}$ be any finite, nonnegative and positive numbers, respectively, for which $\left\|e^{C^{p}}\right\| \leq e^{\left(a^{p}-\lambda^{p} t\right)}, t>0$. Suppose that $\tau_{0}$ is a number satisfying $\tau_{0}>\operatorname{su} p_{p \in \mathcal{P}}\left\{\frac{a^{p}}{\lambda^{p}}\right\}$. For any admissible switching signal $\sigma:[0, \infty) \rightarrow \mathcal{P}$ with $d$ well time no smaller than $\tau_{0}$ the state transition matrix of $C^{\sigma(t)}$ satisfies $\Phi\left(t, t_{0}\right) \leq e^{\left(a-\lambda\left(t-t_{0}\right)\right)}, \forall t \geq t_{0} \geq 0$, where $a=\sup _{p \in \mathcal{P}}\left\{a^{p}\right\}$ and $\lambda=\inf f_{p \in \mathcal{P}}\left\{\lambda^{p}-\frac{a^{p}}{\tau_{0}}\right\}$ (see Morse, 1996, Lemma 2).

Hence, by asking $k>0$ and $k^{2}>\frac{\ell\left(I m\left\{s_{i}\right\}\right)^{2}}{\operatorname{Re}\left\{s_{i}\right\}}$ such that each $C^{\sigma(\mathrm{t})}$ is stable and if the dwell time satisfies the condition $\tau_{0}>\sup _{p \in \mathcal{P}}\left\{\frac{a^{p}}{\lambda^{p}}\right\}$, we can write

$$
\|Y(t)\| \leq e^{\left(a-\lambda\left(t-t_{0}\right)\right)}\|Y(0)\|+\frac{e^{a}}{\lambda}\left(\sup _{0 \leq \tau \leq t}\|\bar{\varepsilon}\|\right),
$$

which implies input-to-state stability of the system (65) with $\bar{\varepsilon}$ as an input. Since $\bar{\varepsilon}$ is globally bounded and converges to zero, $\|Y(t)\|$ is also bounded and converges to zero (see Khalil, 2002, Definition 4.47).

Consequently, we can conclude that $\xi_{i}-\xi_{j} \rightarrow \delta_{i j}$ and $\dot{\xi}_{i} \rightarrow v_{d}(t)$ for all $i, j=1, \ldots, n$. 